\title{
CLOSED LINKAGES
}

\author{
By Colonel R. L. Hipprsley, C.B., R.E.
}

[Received and Read November 9th, 1911.-Received, in Revised form, January 6th, 1912.]

The linkages described in this paper consist of a series of identical three-bar linkages revolving about the same pair of pivots, and formed by repeatedly rotating one of the triangles into which a diagonal divides the quadrilateral about that diagonal, alternate diagonals being used in succession. If, after a given number of such operations, a vertex coincides with its original position, the linkage is said to " close."

The subject has been discussed in an article by Arnold Emch,* who has pointed out the connection between the problem of closing such linkages and the corresponding problem of Poristic Polygons. The treatment is based upon the statement by Darboux + that such a chain of linkages could be formed, and that, if the required coincidence occurred at all, it would occur whatever was the initial position of the quadrilateral. It follows, therefore, that such a compound linkage is poristic. It possesses one degree of freedom, although it fulfils the conditions of a rigid framework.

Darboux has defined three quantities,

$$
p_{12}=a+b-c-d, \quad p_{13}=a-b+c-d, \quad p_{14}=a-b-c+d_{s}
$$

where $a, b, c$, and $d$ are the sides of the quadrilateral ; and has limited his investigations to the case where $p_{12}$ and $p_{13}$ are positive and $p_{14}$ is negative. $t$ Emch has made the same restriction. In the later case the reason is evident ; for it is ouly with this restriction that closed linkages of the sort he had in view can be constructed. But these three quantities may each be either positive or negative; and the object of the present

* Annals of Mathematics, Series 2, Vol. 1 (1900).

$\dagger$ Bulletin des Sciences Mathématiques, 2nd Series, Tome iii, 1879, pp. 120, 121.

$\ddagger$ Darboux, op. cii., pp. 111, 116. 
paper is to remove the restriction and to show that closed linkages of another sort can be constructed whatever the values of $p_{12}, p_{13}$, and $p_{14}$ may be, and that they likewise lead to poristic polygons whose circumscribing and inscribed circles may be partly or wholly external to one another.

Let us consider the side $d$ as fixed. The nature of the motion of the linkage as regards the rotation or oscillation of the various links, and the characteristics of the curve traced out by any point rigidly connected to the opposite side $b$, will depend upon the relative lengths of the links. The case taken by Darboux and by Emch is the one which allows all three of the moving links $a, b, c$ to revolve continuously through complete circles.

If all the permutations of the four quantities $a, b, c$, and $d$ be written out, there will result twenty-four ways in which the order of magnitude of these links can be varied. In each of these there is one critical value of the ratios which permits the three moving links to lie altogether on the line $d$, namely, when the sum of the extremes is equal to the sum of the means. The locus of the carried point has then an extra double point in addition to the three ordinary nodes on the focal circle and to the two triple points at infinity. The curve becomes unicursal, and the elliptic functions by which the variables can be expressed degenerate. The locus is unipartite or bipartite according as one of these sums is greater or less than the other. There are, therefore, forty-eight different species of linkage giving rise to different kinds of curves having special characteristics.

In applying elliptic functions to three-bar motion we have to deal with the following quantities :

$$
\begin{array}{lll}
\alpha_{1}=(b+c)^{2}, & \alpha_{2}=(a+c)^{2}, & \alpha_{3}=(a+b)^{2}, \\
\beta_{1}=(a+d)^{2}, & \beta_{2}=(b+d)^{2}, & \beta_{3}=(c+d)^{2}, \\
\gamma_{1}=(b-c)^{2}, & \gamma_{2}=(a-c)^{2}, & \gamma_{3}=(a-b)^{2}, \\
\delta_{1}=(a-d)^{2}, & \delta_{2}=(b-d)^{2}, & \delta_{3}=(c-d)^{2} .
\end{array}
$$

It is with the relative values of $\alpha, \beta, \gamma$, and $\delta$ that we are concerned. Now, the forty-eight different species of linkage can be grouped into eight classes, each of six species. All the species in any one class exhibit the same peculiarities in the rotation or oscillation of the links, in the number of branches in the locus of the carried point, and in the relative magnitudes of the twelve quantities $\alpha, \beta, \gamma, \delta, \ldots$. The following table gives an analysis of them :- 


\begin{tabular}{|c|c|c|c|c|c|c|c|c|c|}
\hline \multirow{2}{*}{ 总 } & \multirow{2}{*}{ 总总 } & \multirow{2}{*}{ 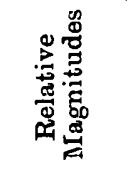 } & \multirow{2}{*}{ 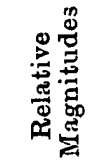 } & \multicolumn{2}{|c|}{$\stackrel{a}{\text { passes } O O^{\prime}}$} & \multicolumn{2}{|c|}{$\stackrel{c}{\text { passes } O O^{\prime}}$} & \multirow{2}{*}{$\begin{array}{l}\text { Links } \\
\text { which } \\
\text { rotate }\end{array}$} & \multirow{2}{*}{$\begin{array}{c}\text { Number } \\
\text { of } \\
\text { Branches }\end{array}$} \\
\hline & & & & ex- ternally & $\begin{array}{l}\text { in- } \\
\text { ternally }\end{array}$ & $\begin{array}{c}\text { ex- } \\
\text { ternally }\end{array}$ & $\begin{array}{l}\text { in- } \\
\text { ternally }\end{array}$ & & \\
\hline 1 & $a_{1} \beta_{1} \gamma_{1} \delta_{1}$ & $\alpha_{2} \beta_{9} \gamma_{2} \delta_{2}$ & $\beta_{3} \alpha_{3} \delta_{3} \gamma$ & yes & no & no & yes & - & 1 \\
\hline 2 & $a_{1} \beta_{1} \gamma_{1} \delta_{1}$ & $\beta_{2} \circ_{2} \delta_{2} \gamma_{2}$ & $a_{3} \beta_{3} \gamma_{3} \delta$ & res & no & yes & no & - & 1 \\
\hline 3 & $a_{1} \beta_{1} \delta_{1} \gamma$ & $a_{2} \beta_{2} \delta_{2} \gamma_{:}$ & $a_{33} \beta_{3} \delta_{33} \gamma$ & yes & yes & yes & yes & $a, b, c$ & 2 \\
\hline 4 & $a_{1} \beta_{1} \delta_{1} \gamma$ & $\beta_{2} a_{2} \gamma_{2} \delta_{2}$ & $\beta_{3} a_{3} \gamma_{3} \delta$ & yes & yes & no & no & $a$ & 2 \\
\hline 5 & $\beta_{1} a_{1} \gamma_{1} \delta$ & $\beta_{2} a_{2} \gamma_{2} \delta$ & $\alpha_{3} \beta_{3} \delta_{3}$ & no & no & yes & yes & $c$ & 2 \\
\hline 6 & $\beta_{1} \alpha_{1} \gamma_{1} \delta$ & $a_{2} \beta_{2} \delta_{2} \gamma_{2}$ & $\beta_{3} \alpha_{3} \gamma_{3}$ & no & no & no & no & $b$ & 2 \\
\hline 7 & $\beta_{1} a_{1} \delta_{1} \gamma$ & $a_{2} \beta_{2} \gamma_{2} \delta_{2}$ & $a_{3} \beta_{3} \gamma_{3}$ & no & yes & yes & no & - & 1 \\
\hline 8 & $\beta_{1} a_{1} \delta_{1} \gamma$ & $\beta_{2} a_{2} \delta_{2} \gamma_{2}$ & $\beta_{3} a_{3} \delta_{3} ?$ & no & yes & no & yes & - & 1 \\
\hline
\end{tabular}

Now, it can easily be seen that $a$ passes $O O^{\prime}$ (Fig. 1) externally if $b+c>a+d$ or $a_{1}>\beta_{1}$, and internally if $a \sim d>b \sim c$ or $\delta_{1}>\gamma_{1}$, and

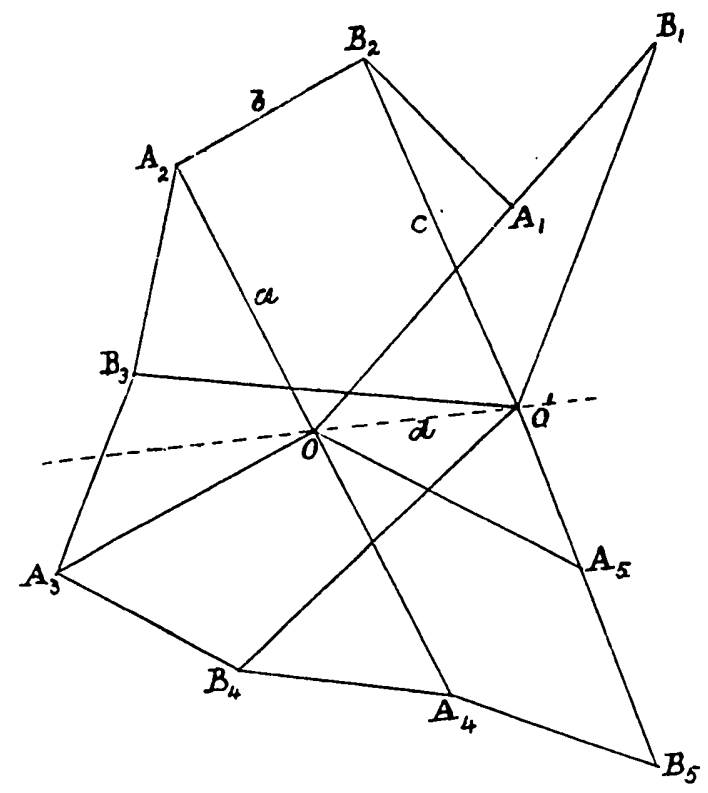

Fic. 1.

therefore revolves if $a_{1}>\beta_{1}>\delta_{1}>\gamma_{1}$. Also $c$ passes $O O^{\prime}$ externally if $a+b>c+d$ or $\alpha_{3}>\beta_{3}$, and internally if $c \sim d>a \sim b$ or $\delta_{3}>\gamma_{3}$, and therefore revolves if $\alpha_{3}>\beta_{3}>\delta_{3}>\gamma_{3}$. Similarly the revolution of $b$ is complete if $\alpha_{2}>\beta_{2}>\delta_{2}>\gamma_{2}$.

Let us consider Class 1. Denote the angles $O^{\prime} O A, O O^{\prime} B$ by $\xi$ and $\eta$. 
The angle $\eta$ cannot increase beyond a certain value given by

$$
\cos \eta=\frac{c^{2}+d^{2}-(a+b)^{2}}{2 c d}
$$

After reaching this value it diminishes. Starting with the linkage in the position when $\eta$ is a maximum, build upon it a series of additional linkages in the manner described. If the last $c$ link falls in the position which is the reflection of $O^{\prime} B_{1}$ in $O O^{\prime}$ or, as shown in Fig. 1, in the position of maximum $\xi$, the linkage will then form one half of a closed linkage. Now superimpose upon this compound linkage another one similar in all respects, but lacking in the first case the last $c$ link and in the second the last $a$ link. Pivot the two linkages together at $O, O^{\prime}$ and $B_{1}$, and either at $B_{5}$ or $A_{5}$, as the case may be. Then move the two $A_{1}$ points in opposite directions, and the whole will open out in the manner shown in Fig. 2.

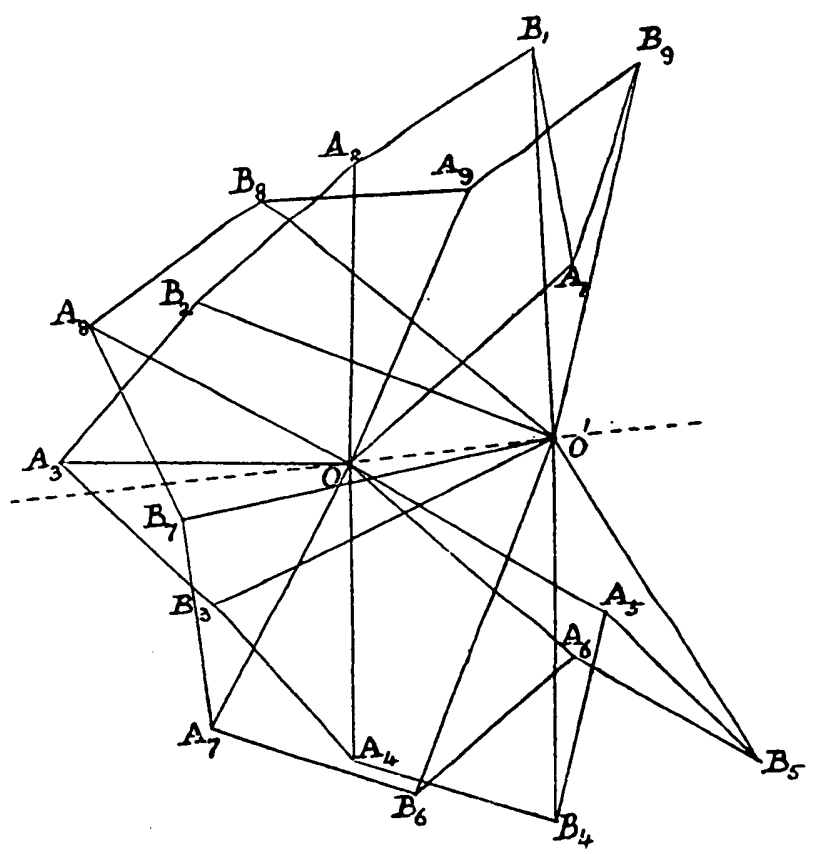

FIg. 2.

This compound linkage may then be regarded as one whole. Any motion imparted to any link will be communicated to all the others, and, when any one $a$ or $c$ link reaches the limit of its motion in one direction, it will turn back and become a part of the superimposed linkage, and will travel in the opposite direction to its other limiting position.

The problem of closing these linkages depends upon a principle pointed 
out by Cayley * and utilized by Darboux. The theorem is in effect that if $x_{1}$ and $x_{2}$ denote the squares of the diagonals $O^{\prime} A_{1}$ and $O^{\prime} A_{2}, x_{1}$ and $x_{2}$ can be expressed in terms of elliptic functions of two arguments $q_{1}$ and $I_{2}$ whose difference is constant. This can be shown in a variety of ways. Perhaps the most natural is derived from

$$
x=a^{2}-2 a d \cos \dot{\xi}+d^{2}=b^{2}-2 b c \cos \omega+c^{2},
$$

where $\omega$ denotes the angle $A B O^{\prime}$. Then

$$
\begin{array}{ll}
\cos \xi=\frac{\beta_{1}-x-\left(x-\delta_{1}\right)}{\beta_{1}-\delta_{1}}, & \cos \omega=\frac{\alpha_{1}-x-\left(x-\gamma_{1}\right)}{a_{1}-\gamma_{1}}, \\
\sin \xi=\frac{2\left\{\left(\beta_{1}-x\right)\left(x-\delta_{1}\right)\right\}_{1}^{\frac{1}{3}}}{\beta_{1}-\delta_{1}}, & \sin \omega=\frac{2\left\{\left(a_{1}-x\right)\left(x-\gamma_{1}\right)\right\}^{3}}{\alpha_{1}-\gamma_{1}} ;
\end{array}
$$

hence $\quad \frac{d \xi_{1}}{\sin \omega_{1}}=\frac{1}{2}\left(\alpha_{1}-\gamma_{1}\right) \frac{d x_{1}}{\left(X_{1}\right)^{\frac{1}{2}}}, \quad \frac{d \xi_{2}}{\sin \omega_{2}}=\frac{1}{2}\left(a_{1}-\gamma_{1}\right) \frac{d x_{2}}{\left(X_{2}\right)^{\frac{1}{2}}}$,

where $\quad X=\left(\alpha_{1}-x\right)\left(\beta_{1}-x\right)\left(x-\gamma_{1}\right)\left(x-\delta_{1}\right)$,

and, as the small displacements of $A_{1}$ and $A_{2}$ are proportional to $\sin \omega_{1}$ and $\sin \omega_{2}$,

$$
d \xi_{1} / \sin \omega_{1}=d \xi_{2} / \sin \omega_{2} ;
$$

hence

$$
\Im_{2}-\Im_{1}=\text { a constant, say } \mu \text {, }
$$

where

$$
\frac{1}{M} \vartheta=\int_{\gamma} \frac{d x}{X^{!}} \quad(a>\beta>x>\gamma>\delta),
$$

and similarly

$$
\Im_{3}-\vartheta_{2}=\mu \text {, }
$$

so that

$$
q_{n}-\vartheta_{1}=(n-1) \mu=4 K-\mu,
$$

since the linkage closes in $4 K$.

Now $\mu$ can be determined from the condition that when

$$
\cos \xi=\frac{(a+b)^{2}-c^{2}+d^{2}}{2 d(a+b)},
$$

$A_{n}$ coincides with $A_{1}, x_{n}=x_{1}$ and $\vartheta_{n}=4 K-\Im_{1}$, so that $2 \Im_{1}=\mu$, and now

$$
x_{1}=a^{2}+d^{2}-\frac{a\left\{(a+b)^{2}-c^{2}+d^{2}\right\}}{a+b}=\frac{\gamma_{1}\left(\beta_{1}-\delta_{1}\right)-\delta_{1}\left(\beta_{1}-\gamma_{1}\right) \operatorname{sn}^{2} g_{1}}{\beta_{1}-\delta_{1}-\left(\beta_{1}-\gamma_{1}\right) \operatorname{sn}^{2} g_{1}},
$$

the modulus being $\frac{\left(\beta_{1}-\gamma_{1}\right)\left(a_{1}-\dot{o}_{1}\right)}{\left(a_{1}-\gamma_{1}\right)\left(\beta_{1}-\delta_{1}\right)}$ which is the same als $\frac{\left(\beta_{2}-\gamma_{2}\right)\left(a_{2}-\delta_{2}\right)}{\left(a_{2}-\gamma_{2}\right)\left(\beta_{2}-\delta_{2}\right)}$; 
from which we obtain

and hence

$$
\operatorname{sn}^{2} \vartheta=\frac{\beta_{2}-\delta_{2}}{a_{2}-\delta_{2}}, \quad \operatorname{cn}^{2} \vartheta=\frac{a_{2}-\beta_{2}}{a_{2}-\delta_{2}}, \quad \operatorname{dn}^{2} \vartheta=\frac{a_{2}-\beta_{9}}{a_{2}-\gamma_{2}} ;
$$

$$
\begin{gathered}
\operatorname{sn} \mu=\frac{2\left\{\left(a_{2}-\gamma_{2}\right)\left(\beta_{2}-\delta_{2}\right)\right\}^{\frac{1}{2}}}{a_{2}+\beta_{2}-\gamma_{2}-\delta_{2}}, \quad \text { cn } \mu= \pm \frac{a_{2}-\beta_{2}-\gamma_{2}+\delta_{2}}{a_{2}+\beta_{2}-\gamma_{2}-\delta_{2}}, \\
\operatorname{dn} \mu= \pm \frac{\alpha_{2}-\beta_{2}+\gamma_{2}-\delta_{2}}{a_{2}+\beta_{2}-\gamma_{2}-\delta_{2}},
\end{gathered}
$$

and the condition that the linkage should close is that $\mu$ must be an aliquot part of $4 K$. Now the 9 that we have just evaluated is the interval between the minimum of $\xi$ and the maximum of $\eta$. This interval is therefore $\frac{1}{2} \mu$; accordingly the interval between the $B_{1}$ and $B_{5}$ of Fig. 1 is $4 \frac{1}{2} \mu$. There is, of course, the same interval from $B_{5}$ back to the starting point. Fig. 2 therefore closes in 9.

The other classes can be dealt with in a similar manner. They naturally resolve themselves into two groups: the first, which includes classes $1 ; 2,7$, and 8 , consist of linkages which give rise to unipartite three-bar curves and which close in $4 K$; the second comprises classes 3 , 4,5 , and 6 , and is composed of those which produce bipartite curves and which close in $2 K$, the motion in the two other elliptic quadrants being represented by the other branch of the curve and being only realizable by an actual disjointing of the mechanism and by its reconstruction in another position. The value of $\mu$ can be obtained by a similar process; for, although it is not always possible to effect the coincidence of the points $A_{n}$ and $A_{1}$ in the manner of the foregoing example, yet, when it is not, it is always possible to make these points occupy positions sym. metrical with respect to $O O^{\prime}$ and where the value of $\cos \xi$ is known. Then $x_{n}=x_{1}$ and $\vartheta_{n}=4 K-\vartheta_{1}$ or $2 K-\vartheta_{1}$ as the case may be, so that in

\begin{tabular}{|c|c|c|c|}
\hline & $\sin ^{2} \mathrm{~S}$ & $\mathrm{cn}^{2} \mathrm{~s}$ & $\mathrm{dn}^{2} \mathrm{~d}$ \\
\hline Classes 1 and $7 \ldots$ & $\frac{\beta_{2}-\delta_{2}}{a_{2}-\delta_{2}}$ & $\frac{a_{3}-\beta_{2}}{a_{2}-\delta_{2}}$ & $\frac{\alpha_{2}-B_{2}}{\alpha_{:}-\gamma_{2}}$ \\
\hline Classes 2 and $8 \ldots$ & $\frac{\beta_{2}-\delta_{2}}{B_{2}-\gamma_{8}}$ & $\begin{array}{l}\delta_{2}-\gamma_{2} \\
\beta_{2}-\gamma_{2}\end{array}$ & $\frac{\delta_{2}-\gamma_{2}}{a_{2}-\gamma_{2}}$ \\
\hline Classes 3 and $6 \ldots$ & $\frac{\beta_{2}-\gamma_{2}}{a_{2}-\gamma_{2}}$ & $\frac{a_{2}-\beta_{2}}{a_{2}-\gamma_{8}}$ & $\frac{a_{2}-\beta_{2}}{a_{2}-\delta_{3}}$ \\
\hline Classes 4 and $5 \ldots$ & $\frac{\alpha_{2}-\delta_{2}}{\beta_{2}-\delta_{9}}$ & $\begin{array}{l}\beta_{2}-a_{2} \\
\beta_{2}-\bar{\delta}_{2}\end{array}$ & $\begin{array}{l}\beta_{2}-\alpha_{2} \\
\beta_{2}-\gamma_{9}\end{array}$ \\
\hline
\end{tabular}
every case $\mu=29$ and can be determined. We shall, in fact, find as follows :- 
which give

\begin{tabular}{|c|c|c|c|}
\hline & $\operatorname{sn} \mu$ & $\operatorname{cn} \mu$ & $\operatorname{dn} \mu$ \\
\hline Classes $1,2,7$ and $8 \ldots$ & $\pm \frac{2\left\{\left(a_{2}-\gamma_{2}\right)\left(\beta_{2}-\delta_{2}\right)\right\}}{a_{2}+\beta_{2}-\gamma_{2}-\delta_{2}}$ & $\pm \frac{a_{2}-\beta_{2}-\gamma_{2}+\delta_{2}}{a_{2}+\beta_{2}-\gamma_{2}-\delta_{2}}$ & $\pm \frac{a_{2}-\beta_{2}+\gamma_{2}-\delta_{3}}{a_{2}+\beta_{2}-\gamma_{2}-\delta_{2}}$ \\
Classes 3,4,5 and $6 \ldots$ & $\pm \frac{2\left\{\left(a_{2}-\delta_{2}\right)\left(\beta_{2}-\gamma_{2}\right)\right\}}{a_{2}+\beta_{2}-\gamma_{2}-\delta_{2}}$ & $\pm \frac{a_{2}-\beta_{2}+\gamma_{2}-\delta_{2}}{a_{2}+\beta_{2}-\gamma_{2}-\delta_{2}}$ & $\pm \frac{a_{2}-\beta_{2}-\gamma_{2}+\delta_{2}}{a_{2}+\beta_{2}-\gamma_{2}-\delta_{2}}$ \\
\hline
\end{tabular}

Now, in Classes $1,2,7$ and 8 , in order to close in $n$ cells, $\mu$ must be equal to $\frac{4 K}{n}$ or 2 to $\frac{2 K}{n}$. If we denote $\frac{1}{\operatorname{sn}^{2} \frac{2 K}{n}}$ by $p$, and $1-\mathrm{dn}^{2} \frac{2 K}{n}$ by $q$, then for classes 1 and 7 ,

or

$$
\frac{a_{2}-\delta_{2}}{\beta_{2}-\delta_{2}}=p, \quad \frac{\beta_{2}-\gamma_{2}}{a_{2}-\gamma_{2}}=q
$$

and

$$
a^{2}-b^{2}+c^{2}-d^{2}+2 a c+2 b d=4 p b d,
$$

$$
-a^{2}+b^{2}-c^{2}+d^{2}+2 a c+2 b d=4 q a c ;
$$

therefore

$$
a c=\frac{p-1}{1-q} b d
$$

and

$$
a^{2}+c^{2}=b^{2}+d^{2}+\frac{2\{p(1-q)-q(p-1)\}}{1-q} b d
$$

$\frac{p(1-q)-q(p-1)}{1-q}$ being $\frac{k^{\prime 2} \operatorname{sn}^{2} \frac{2 K}{n}+\mathrm{cn}^{2} \frac{2 K}{n} \mathrm{dn}^{2} \frac{2 K}{n}}{\operatorname{sn}^{2} \frac{2 K}{n} \mathrm{dn}^{2} \frac{2 K}{n}}$, and always positive.

We can now assign arbitrary values to $b$ and $d$, and obtain from the two foregoing equations the corresponding values of $a$ and $c$, which will ensure closing in $n$ cells. In choosing the values of $b$ and $d$ we must take care to make $a^{2}+c^{2}$ greater than $2 a c$, otherwise $a-c$ will become imaginary. By giving the negative sign to $\left\{(a-c)^{2}\right\}^{3}$ we interchange the values of $a$ and $c$, and the linkage changes to one of the associated class.

For classes 2 and 8 , we put

$$
\frac{\beta_{2}-\gamma_{2}}{\beta_{2}-\delta_{2}}=p, \quad \text { and } \quad \frac{\alpha_{2}-\delta_{2}}{a_{2}-\gamma_{2}}=q ;
$$

which has the effect of simply changing the sign of

$$
\frac{2\{p(1-q)-q(p-1)\}}{1-q} \text {. }
$$


For classes 3 and 6 ,

$$
\begin{aligned}
\operatorname{sn}^{2} \Im & =\frac{1-\operatorname{cn} \frac{2 K}{n}}{1+\operatorname{dn} \frac{2 K}{n}}, \\
\operatorname{dn}^{2} \vartheta & =\frac{\operatorname{cn} \frac{2 K}{n}+\operatorname{dn} \frac{2 K}{n}}{1+\operatorname{cn} \frac{2 K}{n}}, \\
\frac{1}{1-\mathrm{dn}^{2} \Im} & =\frac{1+\operatorname{cn} \frac{2 \pi}{n}}{1-\operatorname{dn} \frac{2 K}{n}},
\end{aligned}
$$

so that, denoting en $\frac{2 K}{n}$ by $r$, and $\operatorname{dn} \frac{2 K}{n}$ by $s$, we get

$$
\begin{gathered}
a c:=\frac{1+s}{1-s} b \iota, \\
a^{2}+c^{2}=l^{2}+d^{2}+\frac{4 r}{1-s} b d .
\end{gathered}
$$

Classes 4 and 5 have the peculiarity that the alternate $a$ links in class 4 and $c$ links in class 5 are moving in opposite directions. If, in class 4 , we consider the motion of $A_{1}, A_{3}, \ldots$ as positive, and that of $A_{2}, A_{4}, \ldots$ as negative, then $\vartheta_{2}, \mathfrak{I}_{4}, \ldots$ are negative arguments, and we get

$$
\begin{aligned}
& \varsigma_{1}+\varsigma_{i 2}=-2 K+\mu, \\
& \Im_{.2}+\varsigma_{3}=-\mu,
\end{aligned}
$$

where $\mu$ is the positive value of $\Im_{2}$ when $\Im_{1}=0$; so that

$$
\begin{aligned}
& \mathfrak{z}_{9}-\mathfrak{\sim}_{1}=2 K-2 \mu, \\
& c_{5}-\Sigma_{3}=2 K-2 \mu \text {, } \\
& \Im_{n+1}-\complement_{n-1}=2 K-2 \mu \text {, }
\end{aligned}
$$

whence

$$
\varsigma_{n+1}-\varsigma_{1}=n h-\mu \mu:
$$

and if $\Im_{n+1}$ coincides with $\check{\sim}_{1}$, that is to say, is $2 K+\complement_{1}$, then

$$
\mu=\frac{n-2}{n} K=K-\frac{2 \pi}{n} \text {; }
$$


from which it is plain that these classes cannot close in an odd number. of cells. Then denoting en $\left(K-\frac{2 K}{n}\right)$ by $r^{\prime}$, and $\mathrm{dn}\left(K-\frac{2 K}{n}\right)$ by $s^{\prime}$, we get

$$
\begin{gathered}
a c=\frac{1-s^{\prime}}{1+s^{\prime}} \dot{b} d, \\
a^{2}+c^{2}=b^{2}+d^{2}-\frac{4 r^{\prime}}{1+s^{\prime}} b l .
\end{gathered}
$$

The tables given in Sir George Greenhill's memoir on "The Third Elliptic Integral and the Ellipsotomic Problem" * afford a ready means of obtaining the values of $p, q, r$, and $s$ for certain values of $n$ in terms of $\Omega$ parameter which may be chosen arbitrarily and which may be considered to be a function of the modulus.

If the links $a$ and $b$ are equal, in the unipartite classes

and

$$
\begin{gathered}
c=\frac{p-1}{1-q} d, \\
a= \pm \frac{(p-q)(p+q-2)}{2(1-q)\{p(1-q)-q(p-1)\}} d ;
\end{gathered}
$$

the upper sign being taken for classes 1 and 7 and the lower for 2 and 8 . Now $p-1,1-q, p-q$ and $p(1-q)-q(p-1)$ are all positive quantities. The possibility of such a linkage depends, therefore, on the sign of $p+q-2$. It can easily be verified that $p+q=2$ when $n=4$. Accordingly a linkage in which $a=b$ is only possible in classes 1 and 7 when $n$ is greater than 4 , and in classes 2 and 8 when $n$ is less than 4 . In classes 3 and 6

$$
c=\frac{1+s}{1-s} d, \quad \text { and } \quad a=\frac{s}{r(1-s)} d ;
$$

and in classes 4 and 5 ,

$$
c=\frac{1-s^{\prime}}{1+s^{\prime}} d, \quad \text { and } \quad a=\frac{s^{\prime}}{r^{\prime}\left(1+s^{\prime}\right)} d ;
$$

all essentially positive as $\mu$ lies in the first elliptic quadrant. There is, therefore, no impossibility in such cases.

With regard to the formation of the related poristic polygons, they can most readily be constructed by drawing tangents to the circle $A_{1} A_{2} \ldots$ through the points $A_{1}, A_{2}, \ldots$. These tangents will intersect on a conic 
whose equation can be determined by eliminating $z$ from

$$
r=\frac{a}{\cos A_{1} O B_{1}}=\frac{2 a^{2} z}{z^{2}+a^{2}-b^{2}}, \quad \text { and } \quad d \cos \theta=\frac{z^{2}-c^{2}+d^{2}}{2 z} ;
$$

where $z$ represents the diagonal $O B_{1}$. This conic becomes a circle if $a=b$. It is only when $a=b$ or $b=c$ that poristic polygons with circumscribing circles can be obtained from a closed linkage. If $a=b$ the conic becomes a circle whose centre is on $O O^{\prime}$ at a distance $\frac{2 a^{2} d}{c^{2}-d^{2}}$ from $O$, and whose radius is $\frac{2 a^{2} c}{c^{2}-d^{2}}$; and this circle is the one into which the circle $B_{1} B_{2} \ldots$ inverts with reference to a circle concentric with, and of twice the radius of, $A_{1} A_{2} \ldots$, but drawn to half the scale. If $b=c$, the tangents through $B_{1}, B_{2}, \ldots$, intersect on a circle whose centre is $\frac{2 c^{2} d}{a^{2}-d^{2}}$ from $O^{\prime}$, and whose radius is $\frac{2 a c^{2}}{a^{2}-d^{2}}$.

Fig. 3 gives an instance of a closed linkage belonging to class 5 , selected because this class produces a poristic polygon inscribed in and circumscribed to circles which are completely external to one another.

It is interesting to note that the polygon $A_{1} A_{2} \ldots$ is always circumscribed to a fixed conic, as also is the polygon $B_{1} B_{2} \ldots$. For Fig. 3 clearly shows that the envelope of $A_{1} A_{2}$ is the reciprocal polar of the locus of $B_{1}$ with respect to the circle $A_{1} A_{2} \ldots$. If the locus of $S_{1}$ is a circle, and $s_{1}, s_{2}, \ldots$ denote the points where the lines $O S_{1}, O S_{2}, \ldots$ again meet the circle, then the polygon $s_{1} s_{2} \ldots$ is similar to the polygon $B_{1} B_{3} \ldots$. For when $a=b, S_{1} O . O s_{1}$ and $S_{1} O . O B_{1}$ are both constant, therefore $O s_{1} / O B_{1}$ is constant. 


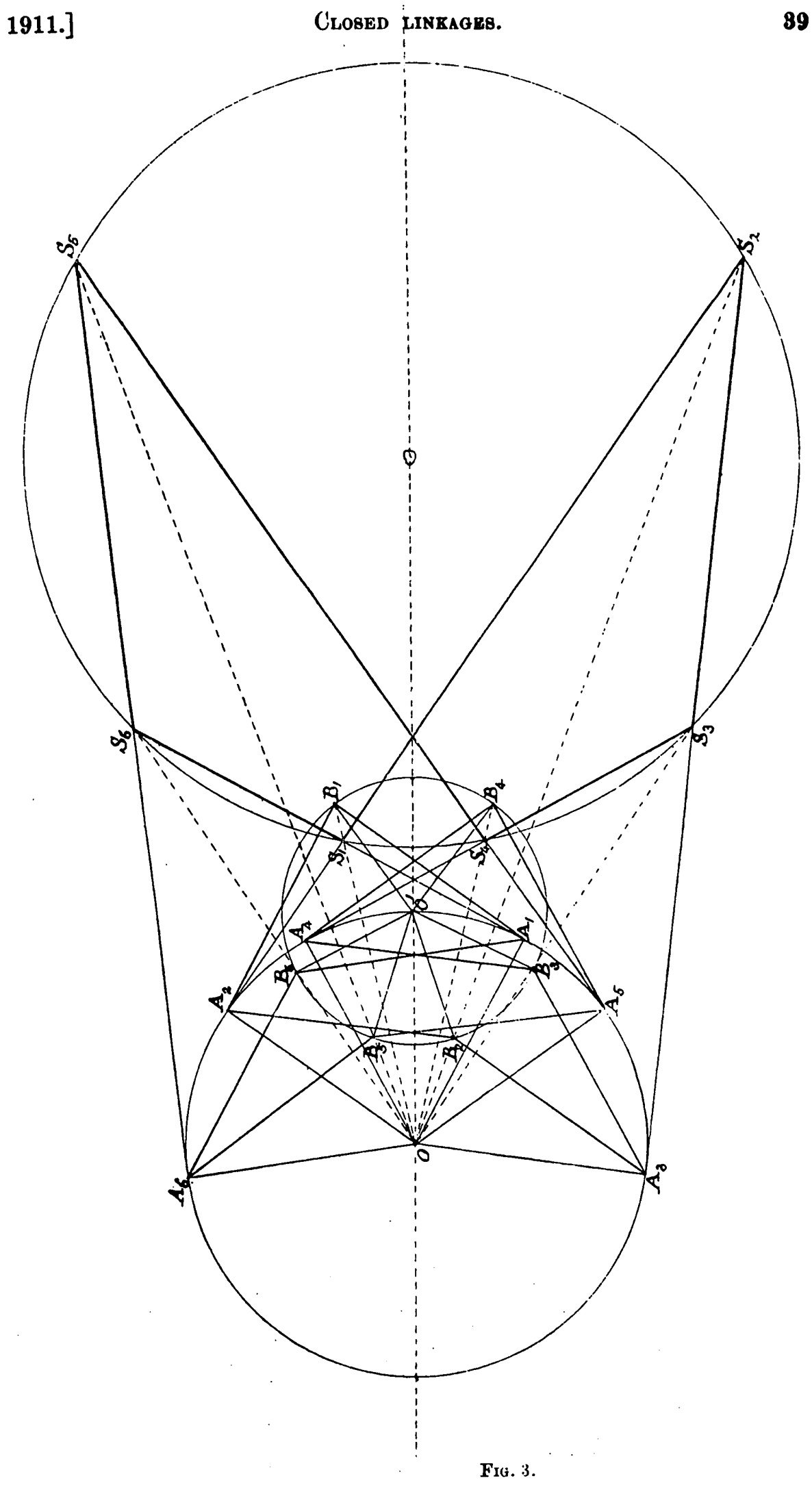

